\title{
Case Report: Mediastinal Teratoma Resembling Vascular Tumor with Rapid Growth Accompanied by Vena Cava Superior Syndrome and Bilateral Paramalignant Pleural Effusion
}

\author{
Ni Made Rini ${ }^{1}$, Suryanti Dwi Pratiwi ${ }^{1}$, Imam Suseno Bayuadi², Dini Rachma Erawati \\ Hendy Setyo Yudhanto 4 \\ ${ }^{1}$ Department of Pulmonology and Respiratory Medicine Medical Faculty of Brawijaya, Dr. Saiful Anwar General Hospital, \\ Malang, Indonesia \\ ${ }^{2}$ Department of Thoracic and Cardiovascular Surgery Medical Faculty of Brawijaya, Dr. Saiful Anwar General Hospital, \\ Malang, Indonesia \\ ${ }^{3}$ Department of Radiology Medical Faculty of Brawijaya, Dr. Saiful Anwar General Hospital, Malang, Indonesia \\ ${ }^{4}$ Department of Anatomical Pathology Medical Faculty of Brawijaya, Dr. Saiful Anwar General Hospital, Malang, \\ Indonesia
}

\begin{abstract}
Background: Teratomas are tumors originate from germ cells, consist of well differentiated tissue from more than one of three layers of embryonic cells: ectoderm, mesoderm and endoderm. Teratomas are generally slow-growing and asymptomatic. Mediastinal teratoma is the most common extra-gonadal germ cell tumor. Superior vena cava syndrome and bilateral paramaligant pleural effusion are complications which can occur in mediastinal teratomas.
\end{abstract}

Case: We report a 37-year-old woman presented with shortness of breath since 1 month before admission, chest pain, cough, weight loss, swolen of face, neck, and right arm. Chest x-ray showed mediastinal tumor. However, no mediastinal tumor had been detected by chest x-ray to examine her heart disease 3 years earlier. Chest computed tomography scan showed anterosuperior mediastinal tumor surrounding great blood vessels and bilateral pleural effusion. Computed tomography guided transthoracic fine needle aspiration biopsy revealed tumor containing mature squamous epithelial component. Patient underwent open biopsy by sternotomy and histological assessment confirmed the diagnosis as mediastinal teratoma with vascular lesions predominantly resembling vascular tumor.

Conclusion: Establishing the diagnosis of mediastinal teratoma is challenging and it requires multi modality approaches.

Keywords: Mediastinal teratoma, vena cava superior syndrome, bilateral paramalignant pleural effusion.

MRJ 2020; 02(01): 68-75

*Corresponding author:

Ni Made Rini (nimaderini89@student.ub.ac.id)

Pulmonology and Respiratory Department, Saiful Anwar Malang Hospital - Universitas Brawijaya, Jaksa

Agung Suprapto St. No. 2, Malang 65112, Indonesia 


\section{Introductuction}

A teratoma is classified a tumor that derives from germ cells. It is composed of welldifferentiated tissues derived from more than one of the all three embryonic cell layers: ectoderm, mesoderm and endoderm. It is usually found both in gonadal organs and at extragonadal sites such as mediastinum, pineal area, sacrococcygeum. ${ }^{1}$

The mediastinum is the extragonadal location in which germ cell tumors are most commonly found. About 5-10\% of all germ cell tumors are found in the mediastinum. In adults, germ cell tumors occur most commonly between the second and fourth decades of life and are found in equal numbers in both sexes. ${ }^{2}$ Teratomas are divited into mature, immature and teratoma with malignant transformation acording to the presence of the different germinal layers (endoderm, mesoderm, and ectoderm). ${ }^{3}$

Mature teratoma of the mediastinum usually grows slowly. The patients are often asymptomatic, and the tumor is found incidentally on chest X-ray. Most of symptoms are related to compression of nearby structures such as chest pain, dyspnea, cough or pulmonary infection. ${ }^{1}$ Mediastina teratoma can compressing the superior vena cava (SVC) and causing superior vena cava (SVC), a collection of clinical signs and symptoms resulting from either partial or complete obstruction of blood flow through the SVC. ${ }^{4}$ Spontaneous rupture into the pleura causing pleural effusion. Mecanissm pleuran effusion related lung or mediastinal tumor can be as paramaligna or malignan pleural effusion. ${ }^{5}$ Mature teratomas can be interpreted as well-defined lobulated masses. However, if there are complications of rupture, it can be a picture of a pleural effusion on a chest $\mathrm{X}$ ray. CT-Scan is the modality of choice in the diagnosis of tumors. Mature teratomas show a multilocular cystic structure in almost $90 \%$ of cases. ${ }^{6}$ Mature teratomas commonly are cystic and possess welldifferentiated tissues from the three germinal cell layers. They often include cartilage or adipose tissue, glandular epithelium, and squamous epithelium. Immature teratomas are less common and contain some mature epithelial and connective tissue components as well as immature areas with neuroectodermal and mesenchymal elements. Most of these tumors are well circumscribed by a wall of fibrous tissue, which may have some calcification within it. Cyst contents may include hair and sebaceous material. ${ }^{2}$ Treatment selection for a given mediastinal tumor depends upon the pathological finding. For the benign, mature teratomas, total resection has been the definitive treatment of choice. ${ }^{7}$ Malignant teratomas sometimes contain other elements, multimodality therapy (surgery + 
chemotherapy + radiotherapy) gives better results. The selection of therapy is based on the elements contained and the patient condition. The management of malignant teratomas with germinal same as seminomas management. Chemotherapy regimens for malignant teratoma include cisplatin, vincristine, bleomycin and methotrexate, etoposide, dactinomycin and cyclophosphamide. $^{8}$

\section{Case}

a 37-year-old woman presented with shortness of breath since 1 month before admission, chest pain, cough, weight loss, swolen of face, neck, and right arm. Chest x-ray in 2017 and 2019 showed mediastinal tumor. However, no mediastinal tumor had been detected by chest x-ray to examine her heart disease 3 years earlier (figure 1, 2,3).

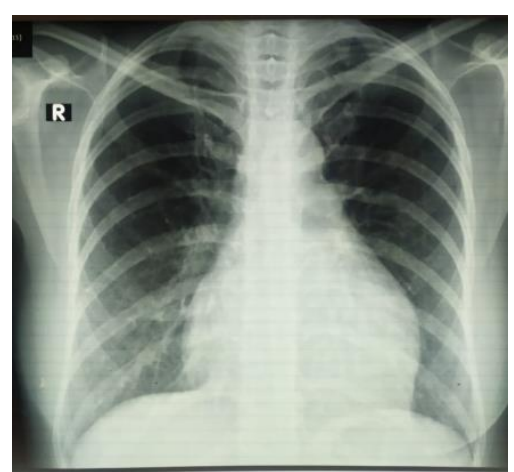

Figure 1. Chest X-ray PA position on May, 13th 2014 showed cardiomegaly and pneumonia, not seen mediastinal masses.

Figure 2. Chest $\mathrm{X}$-ray $\mathrm{PA} /$ lateral $\mathrm{D}$ position on August, 182017 showed bilateral pleural effusion

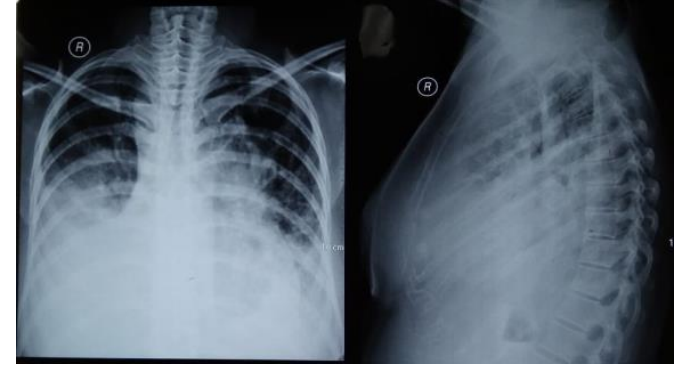

and mediastinal widening suspecetd mediastinal masses.
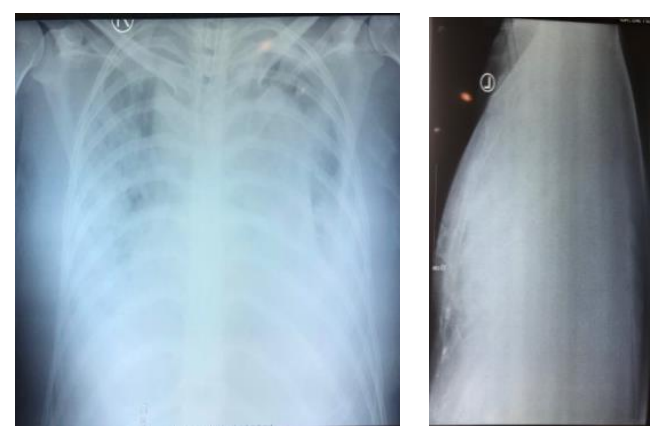

Figure 3. Chest x-ray PA/lateral D position on August, 182017 showed bilateral pleural effusion, edema pulmonum and mediastinal widening suspecetd mediastinal masses

Chest contrast-enhanced CT scan in 2017 revealed a multiloculated isohypodense mass with internal calcifications, intense heterogeneity after the addition of contrast to the anterosuperior mediastinum surrounding and constricting the trachea with the narrowest lumen $\pm 8.8 \mathrm{~mm}$ above the T1 vertebra, covering a. Innominata, a. Left common carotid, a. Left subclavian, superior vena cava, descending aorta, aortic arch, right left main bronchus, pulmonary tract, a. Right and left pulmonary. With conclusion an anterosuperior mediastinal mass with calcification surrounding the trachea and great vessels, a teratoma is suspected. In 2019 a chest CT scan with recontrast was performed, it was found that a multiloculated isohypodense mass with 
calcifications in it, slightly increased after the addition of contrast in the anterosuperior mediastinum measuring \pm $11 \mathrm{~cm} \mathrm{x} 12 \mathrm{~cm} \times 10 \mathrm{~cm}$, the mass covered a. Innominate, a. Bronciocephalic, a. Left communist carotid, a. Left subclavia, superior vena cava, ascending aorta, arcus to descending, trunk pulmonalis, a, pulmonalis right left, and main bronchus right left. A mass is seen constricting the trachea at the level of the T1 vertebra. Conclusion: Mass with calcification in the anterosuperior mediastinum covering large vasculature, relatively fixed size (Figure $4,5)$.

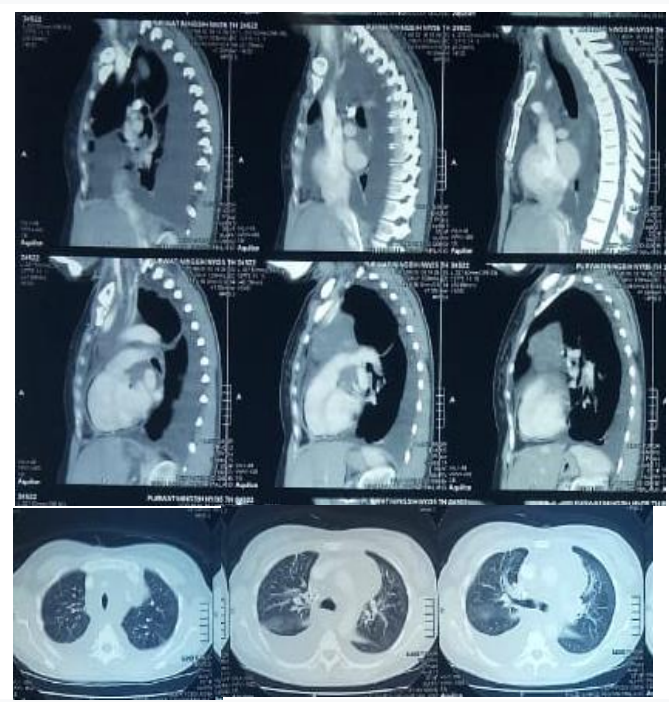

Figure 4. Chest contrast-enhanced CT scan on September, 6th 2017 showed Anterosuperior mediastinal mass with calcification surrounding the trachea and great vessels, suspected teratoma, Bilateral pleural effusion, Bronchitis, Thoracolumbar spondylosis.

Figure 5. Chest contrast-enhanced CT scan on February, 14 th 2019 showed mass with calcification in the anterosuperior mediastinum enclosing large vasculature relatively fixed size, multiple lymphadenopathy in right lower paratrachea, left upper paratrachea, subcarina, with largest engraving

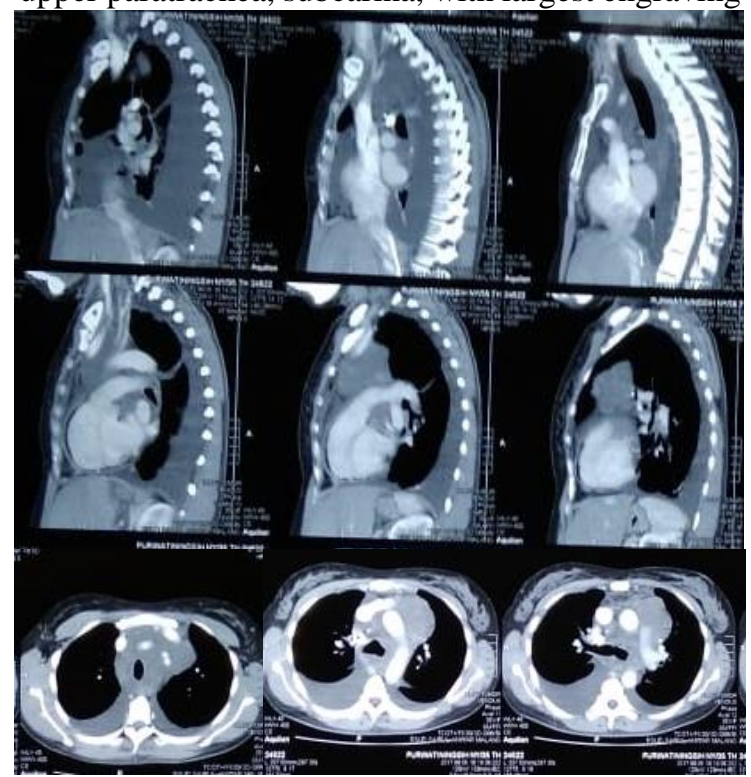

in left paratrachea increase in size, multiple nodules with infiltrates on bilateral lung ec suspected metastatic process new lesion, bilateral pleural effusion, and ascites.

Citology from trans thoracal needal aspiration (TTNA) revealed an anterior mediastinal mass containing a mature squamous epithelial component (Figure 6). The patient underwent thoracotomy open biopsy, histology was found tissue consists of connective tissue, fat tissue, muscle and various blood vessels with bleeding area. No malignancy was found in the preparation. With conclussion may represent part of a teratoma with a predominant vascular lesion (figure 7). 

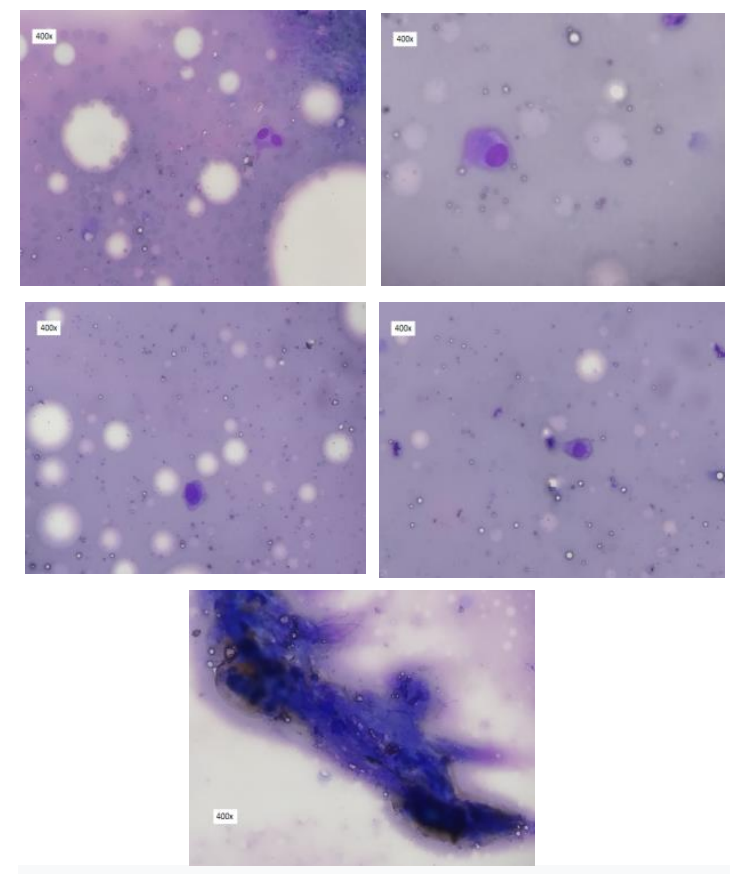

Figure 6. Citological from TTNA showed Tumors containing a mature squamous epithelial component.

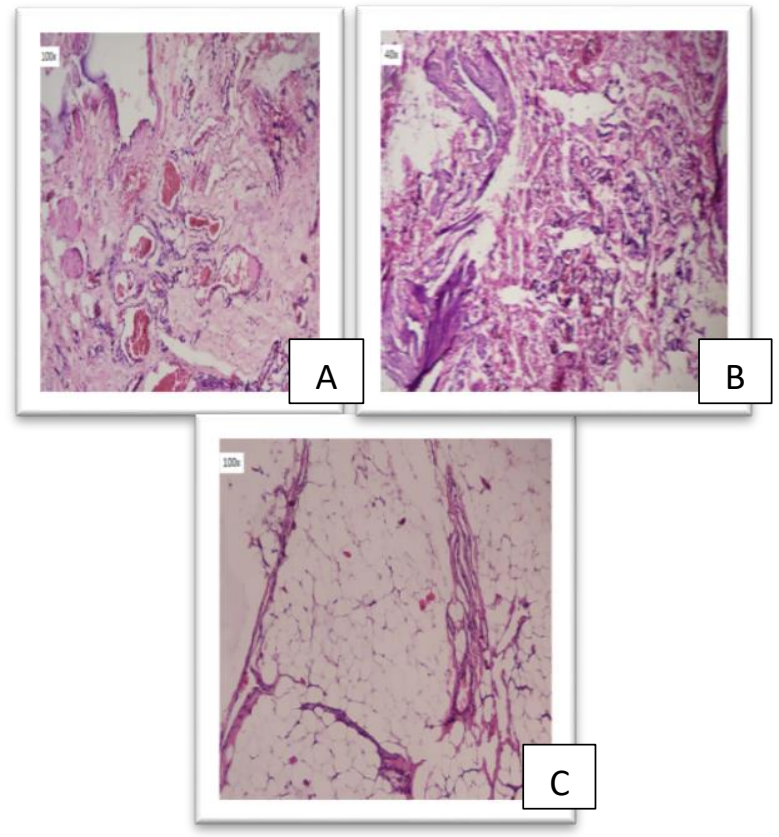

Figure 7. Histological from open biopsy showed (A) dominant blood vessels , (B) skeletal muscle, and (C) fat.

The patient had swelling of the face, neck, and right arm, increased neck venous pressure and venectation of the chest were found. Chest CT scan revealed an anterior mediatinal mass surrounding the superior vena cava, this is a symptom and sign of superior vena cava syndrome.

The patient was complain about shortness of breath, percussion revealed dullness and decreased of breath sounds on auscultation in the middle and lower of the both side of the chest. The chest $\mathrm{x}$-ray and chest CT sacan showed bilateral pleural effusion without rupture of the mass. Pleural fluid analysisis showed transudate and no malignant cells were found on pleural fluid cytology.

Based on the anamnesis, physical examination, and other testing the patiend was diagnosed with mediastinal teratoma resembling vascular tumor with rapid growth accompanied by vena cava superior syndrome and bilateral paramalignant pleural effusion.

\section{Discussion}

This case was reported a 37-year-old woman with mediastinal teratoma resembling vascular tumor with rapid growth accompanied by vena cava superior syndrome and bilateral paramalignant pleural effusion.

Cases of teratoma in adults, occurring most frequently between the second and fourth decades of life and found in equal numbers 
in both sexes. Epidemiologically, this case is in accordance with the literatur. ${ }^{2}$

The patient complain about shortness of breath since 1 month before admission, chest pain, cough, weight loss, swolen of face, neck, and right arm. Most teratomas are asymptomatic. However, as the size of the teratoma increases, it can cause various symptoms. Symptoms that can be found are coughing due to compression of the trachea or bronchi, or erosion of the airway chest pain, arising from tension in mediastinal tissue, tissue invasion, or bone erosion, and shortness of breath. ${ }^{1}$

Chest CT scan revealed a multiloculated isohypodense mass with internal calcifications, which became heterogeneous after adding contrast to the anterosuperior mediastinum surrounding and constricting the trachea and great vessels. Conclusion an anterosuperior mediastinal mass with calcification surrounding the trachea and great vessels, a teratoma is suspected. The location of the tumor in this patient is in accordance with the literature predilection that mediastinal teratomas grow mostly in the anterior mediastinum. The CT-Scan picture is a typical picture of a teratoma mass, namely a cystic mass, well-defined, regular edges and calcified. ${ }^{6}$

Histology from trans thoracal needal aspiration (TTNA) revealed an anterior mediastinal mass containing a mature squamous epithelial component. Since teratoma consists of several germ cells, sitology examination from trans thoracal needal aspiration (TTNA) it is difficult to identify the teratoma because some cells are not collected during the aspiration. The diagnosis can be made when other types of cells are also found. The diagnosis of a teratoma on a small cytological or biopsy specimen should be made only after radiographic observations and serological studies are performed that can exclude mixed germ cell tumour. ${ }^{6}$

Large tumors, particularly in the mediastinum, can be highly vascularized and complicating surgical resection. The blood supply to the mediastinal mass is highly variable. These large feeding vessels can cause technical complications and bleeding during surgical resection. In conditions of compression or invasion of the vena cava by mediastinal tumors, venous circulation will be maintained through collateral veins which can also cause significant surgical bleeding complications. ${ }^{9}$ During and after surgery there was bleeding complication in this patient.

Histology was found tissue consists of connective tissue, fat tissue, muscle and various blood vessels with bleeding area. No malignancy was found in the preparation. With conclussion may represent part of a teratoma with a 
predominant vascular lesion. The predominant vascular lesion may be the cause of severe bleeding during surgery. The challenge in establishing the diagnosis of teratoma is in taking specimens, especially with the small biopsy technique where the risk for errors in interpreting the cell types in the specimen will be high.To avoid this problem, careful analysis of clinical, histopathological, and radiographic findings is necessary. The best way to take a specimen is to remove all of the tumor, that is, by total resection which can also be used as a treatment. ${ }^{10}$

Patien severe from complication such as superior vena cava syndrome and bilateral pleura effusion. Intrathoracal masses involving the superior vena cava can cause superior vena cava syndrome. ${ }^{4}$ Pleural fluid analysisis showed transudate and no malignant cells were found on pleural fluid cytology. Pleural effusion in teratoma can occur through several mechanisms, including rupture of the teratoma mass, implantation of tumor cells on the pleural surface, pleurisy caused by pneumonitis secondary to the tumor, due to obstruction of the flow of lymph or blood vessels, erosion of blood vessels or lymph so that the formation of pleural fluid increases. , direct invasion of the tumor into the pleural cavity through the thoracic wall. ${ }^{11}$ Paramalignant pleural effusions are found in patients with solid tumors without direct pleural involvement and no evidence of malignant cells in the pleural fluid, often transudate with normal glucose levels. ${ }^{5}$

\section{Conslusion}

Mediastinal teratoma with rapid growth is a rare case and can cause complications such as vena cava superior syndrome and bilateral paramalignant pleural effusion. Establishing the diagnosis of mediastinal teratoma is challenging and requires multimodality approach.

\section{Reference}

1. No, T.H., Seol, S.H., Seo, G.W., Kim, D.I., Yang, S.Y., Jeong, C.H., Hwang, Y.H., \& Kim, J.Y. 2015. Benign Mature Teratoma in Anterior Mediastinum. Journal of clinical medicine research, 7(9), 726-728.

2. Mueller, D.K. 2017. Teratomas and Other Germ Cell Tumors of the Mediastinum. Emedicine Medscape. https://emedicine.medscape.com/articl e/427395-overview.

3. Paul, S.S. 2011. Diseases of the testis and epididymis. Clinical Ultrasound (Third Edition). 593-620.

4. Seligson, M.T. and Surowiec, S.M. 2021. Superior Vena Cava Syndrome. StatPearls Publishing. https://www.ncbi.nlm.nih.gov/books/ NBK441981/

5. Gurung, P., Goldblatt, M.R., Huggins, J.T., Doelken, P., Sahn, S.A. 2009. 'Pleural Fluid Characteristics of Paramalignant Effusion', Chest. The American College of Chest Physicians, 136(4),p.44S.

6. Mandal, G., Bhattacharya, S., Dey, A., Kar, S., Saha, S. 2016. Mature cystic teratoma of mediastinum with pleural effusion: An uncommon entity. Nigerian Posgratuate Medical Journal. 23(1):41-43. 
7. PDPI. 2003. Tumor Mediastinum (Tumor Mediastinum Nonlimfoma), Pedoman Diagnosis dan

Penatalaksanaan di Indonesia. Jakarta.

8. Ibarra-Perez, C. et al. 2017. Benign and Malignant Germ Cell Tumors of the Mediastinum. General Thoracic Surgery, Edition: Eighth edition, Chapter: 167.

9. Li, W. W., van Boven, W. J., Annema, J. T., Eberl, S., Klomp, H. M., \& de Mol, B. A. 2016. Management of large mediastinal masses: surgical and anesthesiological considerations. Journal of thoracic disease, 8(3), E175-E184.

10. Kesler, K.A., Rieger, K.M., Hammoud, Z.T., Kruter, L.E., Perkins, S.M, Turrentine, M.W., et al 2008. A 25year single institution experience with surgery for primary mediastinal nonseminomatous germ cell tumors. Ann Thorac Surg, 85(2):371-8.

11. Porcel, J.M. 2019. Diagnosis and characterization of malignant effusions through pleural fluid cytological examination. Current opinion in pulmonary medicine, 25(4), pp.362368. 